\title{
Stabilization of a Predator-Prey System with Nonlocal Terms
}

\author{
S. Aniţa* \\ Faculty of Mathematics, "Alexandru Ioan Cuza" University of Iaşi, Iaşi 700506, Romania \\ "Octav Mayer" Institute of Mathematics of the Romanian Academy, Iaşi 700506, Romania
}

\begin{abstract}
We investigate the zero-stabilizability for the prey population in a predator-prey system via a control which acts in a subregion $\omega$ of the habitat $\Omega$, and on the predators only. The dynamics of both interacting populations is described by a reaction-diffusion system with nonlocal terms describing migrations. A necessary condition and a sufficient condition for the zero-stabilizability of the prey population are derived in terms of the sign of the principal eigenvalues to certain non-selfadjoint operators. In case of stabilizability, a constant stabilizing control is indicated. The rate of stabilization corresponding to such a stabilizing control is dictated by the principal eigenvalue of a certain operator. A large principal eigenvalue leads to a fast stabilization to zero of the prey population. A method to approximate all these principal eigenvalues is presented. Some final comments concerning the relationship between the stabilization rate and the properties of $\omega$ and $\Omega$ are given as well.
\end{abstract}

Keywords and phrases: zero-stabilizability, state constraints, comparison result, principal eigenvalue, population dynamics, reaction-diffusion system

Mathematics Subject Classification: 35B35, 35B09, 35B51, 92D25, 35K57

\section{Problem formulation}

Let $\Omega \subset \mathbf{R}^{N} N \in\{2,3\}$ be a bounded domain (open and connected), $\omega \subset \subset \Omega$ (i.e., $\bar{\omega} \subset \Omega$ ) an open subset, both with sufficiently smooth boundaries $\partial \Omega$ and $\partial \omega$, respectively, and such that $\Omega \backslash \bar{\omega}$ is a domain. Consider a reaction-diffusion system with nonlocal terms that describes the dynamics of two interacting populations (prey and predator) which live in the same habitat $\Omega$ and are subject to a control

${ }^{*}$ Corresponding author. E-mail: sanita@uaic.ro 
$u$ that acts in the subset $\omega$ :

$$
\left\{\begin{array}{rlrl}
\partial_{t} h(x, t)- & d \Delta h(x, t)=r(x) h(x, t)\left(1-\frac{h(x, t)}{K(x)}\right)+\int_{\Omega} k_{1}\left(x, x^{\prime}\right) h\left(x^{\prime}, t\right) d x^{\prime} & \\
& -F(h(x, t)) p(x, t), & & (x, t) \in Q_{0} \\
\partial_{t} p(x, t)= & -a(x) p(x, t)+c \int_{\Omega} k_{2}\left(x, x^{\prime}\right) F\left(h\left(x^{\prime}, t\right)\right) p\left(x^{\prime}, t\right) d x^{\prime} & \\
& +\chi_{\omega}(x) u(x, t), & (x, t) \in Q_{0} \\
\partial_{\nu} h(x, t)= & 0, & (x, t) \in \Sigma_{0} \\
h(x, 0)=h_{0}(x), p(x, 0)=p_{0}(x), & & x \in \Omega,
\end{array}\right.
$$

where $\chi_{\omega}$ is the characteristic function of $\omega, Q_{T}=\Omega \times(T,+\infty), \Sigma_{T}=\partial \Omega \times(T,+\infty)$, and $\partial_{\nu}$ denotes the normal derivative. Here $h(x, t)$ is the density of the prey population species and $p(x, t)$ is the density the predator population species at position $x$ and moment $t$. The natural growth rate of the prey population is $r(x)$ and the natural decay rate of the predator population is $a(x)$ at position $x$. A ratio $\tilde{c}$ of the prey population may diffuse and the ratio $1-\tilde{c}$ of it migrates. If the diffusion rate in the absence of the migration is $\tilde{d}$ and the proportion of preys emigrating from $x$ to other locations in the absence of diffusion is $\tilde{w}(x)$, then we denote $d=\tilde{c} \tilde{d}$ and $w(x)=(1-\tilde{c}) \tilde{w}(x)$, and we get that the population immigrated at position $x$ is $\int_{\Omega} k_{1}\left(x, x^{\prime}\right) h\left(x^{\prime}, t\right) d x^{\prime}$, where $k_{1}\left(x, x^{\prime}\right)=l\left(x, x^{\prime}\right) w\left(x^{\prime}\right)$ and $l\left(x, x^{\prime}\right)$ gives the proportion of preys immigrating to $x$ from location $x^{\prime}$. The prey population diffuses in the whole habitat, while the predator population does not. $\frac{h(x, t)}{K(x)}$ represents a logistic term $(K(x)$ being the carrying capacity of the habitat for the prey population) and the term $\int_{\Omega} k_{1}\left(x, x^{\prime}\right) h\left(x^{\prime}, t\right) d x^{\prime}$ describes the immigration of the prey population at position $x$ and moment $t$. We have denoted by $F(h)$ the functional response to predation, see [5], [14], [15]. The prey captured at location $x^{\prime}$ and moment $t$ is transformed into biomass via the conversion factor $c$ giving a numerical response to predation $c F\left(h\left(x^{\prime}, t\right)\right) p\left(x^{\prime}, t\right)$, this quantity being distributed over $\Omega$ via the kernel $k_{2}$ which must respect the total biomass conservation. We assume there is no population exchange through the boundary of the habitat. The control $u \in U=\left\{v \in L^{\infty}(\omega \times(0,+\infty)) ; 0 \leq v(x, t) \leq L\right.$ a.e. $\}$ $(L \in(0,+\infty))$, represents the density of an infusion of predators in the subdomain $\omega$. The initial densities of prey and predator populations are $h_{0}$ and $p_{0}$, respectively.

We shall work under the following hypotheses:

$$
\begin{gathered}
d, c \in(0,+\infty), \quad r, K, a, h_{0}, p_{0} \in L^{\infty}(\Omega), \quad k_{1}, k_{2} \in L^{\infty}(\Omega \times \Omega) ; \\
r(x) \geq r_{0}, \quad K(x) \geq K_{0}, \quad a(x) \geq a_{0},
\end{gathered}
$$

where $r_{0}, K_{0}, a_{0}$ are positive constants;

$$
\begin{gathered}
k_{1}\left(x, x^{\prime}\right) \geq 0, k_{2}\left(x, x^{\prime}\right) \geq 0 \quad \text { a.e. in } \Omega \times \Omega, \quad \int_{\Omega} k_{2}\left(x, x^{\prime}\right) d x=1 \quad \text { a.e. } x^{\prime} \in \Omega ; \\
h_{0}(x) \geq 0, p_{0}(x) \geq 0 \quad \text { a.e. in } \Omega, \quad\left\|h_{0}\right\|_{L^{\infty}(\Omega)}>0 .
\end{gathered}
$$

Function $F:[0,+\infty) \rightarrow[0,+\infty)$ has one of the following forms: $F(h)=\rho h, F(h)=\frac{\rho h^{k}}{1+q h^{k}}(\rho, q \in$ $(0,+\infty)$, and $k$ is a positive integer), corresponding to Lotka-Volterra or Holling $k+1$ functional response to predation. Remark that in all cases, $F$ is continuously differentiable, strictly increasing and $F(0)=0$. For other population models with nonlocal terms we refer to [8], [10].

Our goal is to stabilize to zero the prey population via an infusion of predators in the subdomain $\omega$. It corresponds to real situations when it is very difficult to act directly on the prey population. Such a problem is totally justified if we view for example the preys as an insect herbivore population (a pest) and the predators as a population of parasitoids. The Lotka-Voltera and the Holling II functional response to predation correspond to a specialized parasitoid while the Holling $k+1$, with $k \geq 2$, corresponds to a generalist parasitoid population. Another example is that of a damaging herbivore population and a population of carnivores. This is a stabilization problem with an indirect control (we act on the preys via the predators) and state constraints (both population densities must be nonnegative). 
Existence and uniqueness of a solution $\left(h^{u}, p^{u}\right)$ to $(1.1)$ as well as the nonnegativity of its components, i.e.

$$
h^{u}(x, t) \geq 0, \quad p^{u}(x, t) \geq 0 \quad \text { a.e. in } Q_{0}
$$

follow as in [2], [3], [4], [5].

Definition 1.1. We say that the prey population is zero-stabilizable if for any $h_{0}, p_{0}$ satisfying the hypotheses there exists $u \in U$ such that

$$
\lim _{t \rightarrow+\infty} h^{u}(\cdot, t)=0 \quad \text { in } L^{\infty}(\Omega) .
$$

Remark that we are dealing here with stabilization with control constraints and with state constraints. Actually, for any $u \in U, h^{u}$ and $p^{u}$ are nonnegative. For stabilization of diffusive systems without constraints we refer to [13], while for some stability results concerning a non local reaction-diffusion equation see [18].

We will show that there is a deep relationship between the zero-stabilizability and the sign of $\lambda_{1}(\omega, \gamma)$, the principal eigenvalue of

$$
\begin{cases}-d \Delta \varphi(x)-r(x) \varphi(x)-\int_{\Omega} k_{1}\left(x, x^{\prime}\right) \varphi\left(x^{\prime}\right) d x^{\prime}+\gamma P(x) \varphi(x)=\lambda \varphi(x), & x \in \Omega \\ \partial_{\nu} \varphi(x)=0, & x \in \partial \Omega,\end{cases}
$$

where $P(x)=\frac{L \chi_{\omega}(x)}{a(x)}$ a.e. in $\Omega$ and $\gamma \in[0,+\infty)$.

The present paper continues the investigations in [1], [2], [3], [4], [5]. The main difference is that in the present paper we intend to erradicate the prey population by increasing the number of predators (in the previous papers the control was done by decreasing the number of prey or by decreasing the number of predators). Since we cannot use the same comparison results for reaction-diffusion systems as in the previous papers we have to consider here a different approach.

Here is the plan of the paper. Section 2 concerns a necessary condition for the zero-stabilization of the prey population in terms of the sign of the principal eigenvalue of a certain non-selfadjoint operator. A sufficient condition for the nonnegative zero-stabilizability, also in terms of the sign of the principal eigenvalue of another non-selfadjoint operator is given in the next section. A large principal eigenvalue leads to a fast stabilization to zero via a constant control. A method to approximate the above mentioned principal eigenvalues is indicated in Section 4. Some final comments concerning the relationship between the stabilization rate and the properties of $\omega$ and $\Omega$ are given in Section 5 .

\section{A necessary condition for the zero-stabilization of the prey population}

The existence of $\lambda_{1}(\omega, \gamma)$ (for any $\gamma \in \mathbf{R}$ ) and their basic properties follow via lemma 2.1 in [3]. Their proofs are based on Krein-Rutman's theorem [9]).

Here is the necessary condition for the zero-stabilizability of the prey (pest) population:

Theorem 2.1. If the prey population is zero-stabilizable then $\lambda_{1}\left(\omega, F^{\prime}(0)\right) \geq 0$.

Proof. Assume that for any initial data $h_{0}, p_{0}$ satisying the hypotheses there exists a stabilizing control $u$. Since $\lim _{t \rightarrow+\infty} h^{u}(\cdot, t)=0$ in $L^{\infty}(\Omega)$, it follows that for any $\varepsilon>0$, there exists $t_{1}(\varepsilon) \in(0,+\infty)$ such that

$$
0 \leq h^{u}(x, t), F\left(h^{u}(x, t)\right)<\varepsilon \quad \text { a.e. } x \in \Omega,
$$

for any $t \geq t_{1}(\varepsilon)$.

Integrating over $\Omega$ both terms in the equation satisfied by $p^{u}$ we obtain that

$$
\frac{d}{d t}\left(\int_{\Omega} p^{u}(x, t) d x\right) \leq-\int_{\Omega} a(x) p^{u}(x, t) d x+\operatorname{Lmeas}(\omega)+\varepsilon c \int_{\Omega} p^{u}(x, t) d x
$$


(we have used that $\int_{\Omega} k_{2}\left(x, x^{\prime}\right) d x=1$ a.e. $x^{\prime} \in \Omega$ )

$$
\leq-a_{0} \int_{\Omega} p^{u}(x, t) d x+\operatorname{Lmeas}(\omega)+\varepsilon c \int_{\Omega} p^{u}(x, t) d x,
$$

for any $t \geq t_{1}(\varepsilon)$. Integrating this ordinary differential inequality we conclude that

$$
\limsup _{t \rightarrow+\infty} \int_{\Omega} p^{u}(x, t) d x \leq \frac{\operatorname{Lmeas}(\omega)}{a_{0}-c \varepsilon},
$$

for sufficiently small $\varepsilon>0$, and consequently the mapping $t \mapsto \int_{\Omega} p^{u}(x, t) d x$ is bounded on $[0,+\infty)$ (i.e., there exists $M \in[0,+\infty)$ such that $0 \leq \int_{\Omega} p^{u}(x, t) d x \leq M$, for any $\left.t \in[0,+\infty)\right)$.

Using a comparison result for the solutions to ordinary differential equations we get that

$$
p^{u}(x, t) \leq P_{\varepsilon}(x, t) \quad \text { a.e. in } \Omega \times\left(t_{1}(\varepsilon),+\infty\right),
$$

where $P_{\varepsilon}$ is the solution to

$$
\begin{cases}\partial_{t} P(x, t)=-a(x) P(x, t)+\varepsilon c K_{2} M+L \chi_{\omega}(x), & (x, t) \in Q_{t_{1}(\varepsilon)} \\ P\left(x, t_{1}(\varepsilon)\right)=p^{u}\left(x, t_{1}(\varepsilon)\right)+1, & x \in \Omega,\end{cases}
$$

where $K_{2} \in(0,+\infty)$ satisfies $0 \leq k_{2}\left(x, x^{\prime}\right) \leq K_{2}$ a.e. in $\Omega \times \Omega$. Since

$$
\lim _{t \rightarrow+\infty} P_{\varepsilon}(\cdot, t)=\frac{L \chi_{\omega}+\varepsilon c K_{2} M}{a} \text { in } L^{\infty}(\Omega)
$$

we may infer that

$$
\limsup _{t \rightarrow+\infty} p^{u}(\cdot, t) \leq \frac{L \chi_{\omega}+\varepsilon c K_{2} M}{a} \text { in } L^{\infty}(\Omega)
$$

for sufficiently small $\varepsilon>0$. So, for any such $\varepsilon$, there exists $t_{2}(\varepsilon) \geq t_{1}(\varepsilon)$ such that

$$
p^{u}(x, t) \leq \frac{L \chi_{\omega}}{a}+\varepsilon \quad \text { a.e. in } Q_{t_{2}(\varepsilon)} .
$$

This yields: there exists $t_{3}(\varepsilon) \geq t_{2}(\varepsilon)$ such that

$$
0 \leq F\left(h^{u}(x, t)\right) \leq\left(F^{\prime}(0)+\varepsilon\right) h^{u}(x, t) \text { a.e. in } Q_{t_{3}(\varepsilon)},
$$

for sufficiently small $\varepsilon>0$.

Since $h^{u}$ satisfies

$$
\partial_{t} h^{u}(x, t)-d \Delta h^{u}(x, t) \geq r(x) h^{u}(x, t)-\frac{r(x) \varepsilon}{K(x)} h^{u}(x, t)+\int_{\Omega} k_{1}\left(x, x^{\prime}\right) h\left(x^{\prime}, t\right) d x^{\prime}-\left(\frac{L \chi_{\omega}}{a}+\varepsilon\right) F\left(h^{u}(x, t)\right)
$$

a.e. in $Q_{t_{2}(\varepsilon)}$, we may infer that there exists $c_{2} \in(0,+\infty)$ such that

$$
\partial_{t} h^{u}(x, t)-d \Delta h^{u}(x, t) \geq r(x) h^{u}(x, t)+\int_{\Omega} k_{1}\left(x, x^{\prime}\right) h\left(x^{\prime}, t\right) d x^{\prime}-\frac{L \chi_{\omega}}{a} F^{\prime}(0) h^{u}(x, t)-c_{2} \varepsilon h^{u}
$$

a.e. in $Q_{t_{3}(\varepsilon)}$, for sufficiently small $\varepsilon>0$.

Since the equation satisfied by $h^{u}$ may be viewed as a parabolic equation

$$
\partial_{t} h-d \Delta h=\tilde{r} h+G(x, t),
$$

with $G(x, t) \geq 0$ in $Q_{0}$ and $h_{0}$ satisfies the hypotheses, we may conclude that $h^{u}(x, t)>0$ a.e. in $Q_{0}$. 
Using a comparison result for integro-partial differential equations ([3], lemma A.3; for comparison principles for parabolic equations without nonlocal terms we refer to [16]) we get that

$$
0 \leq H_{\varepsilon}(x, t) \leq h^{u}(x, t) \quad \text { a.e. in } Q_{t_{3}(\varepsilon)}
$$

(for sufficiently small $\varepsilon>0$ ), where $H_{\varepsilon}$ is the solution to

$$
\left\{\begin{array}{rlrl}
\partial_{t} H(x, t)-d \Delta H(x, t)=r(x) H(x, t)+\int_{\Omega} k_{1}\left(x, x^{\prime}\right) H\left(x^{\prime}, t\right) d x^{\prime} & \\
\quad-\frac{L \chi_{\omega}(x)}{a(x)} F^{\prime}(0) H(x, t)-c_{2} \varepsilon H(x, t), & & (x, t) \in Q_{t_{3}(\varepsilon)} \\
\partial_{\nu} H(x, t)=0, & & (x, t) \in \Sigma_{t_{3}(\varepsilon)} \\
H\left(x, t_{3}(\varepsilon)\right)=h^{u}\left(x, t_{3}(\varepsilon)\right), & x \in \Omega .
\end{array}\right.
$$

We may infer that

$$
\lim _{t \rightarrow+\infty} H_{\varepsilon}(\cdot, t)=0 \quad \text { in } L^{\infty}(\Omega)
$$

and in conclusion (using the positivity of $h^{u}\left(x, t_{3}(\varepsilon)\right)$ ) we obtain (via theorem 2.3 in [3]) that $\lambda_{1}\left(\omega, F^{\prime}(0)\right)+$ $c_{2} \varepsilon>0$ for sufficiently small $\varepsilon>0$, and finally that $\lambda_{1}\left(\omega, F^{\prime}(0)\right) \geq 0$.

Remark 2.2. If $F$ corresponds to a Holling $k+1$ functional response to predation, with $k \geq 2$, then the predators considered here are generalist. It follows immediately that $F^{\prime}(0)=0$ and consequently $\lambda_{1}\left(\omega, F^{\prime}(0)\right) \leq-r_{0}<0$. The conclusion is that using an infusion of generalist predators we cannot stabilize to zero (erradicate) the prey (pest) population; when the prey population is small, the predators focus on other prey population species. The infusion will only diminish below a certain level the prey population.

Remark 2.3. If the predator population diffuses in the habitat $\Omega$, then the second equation in (1.1) must be replaced by

$$
\partial_{t} p(x, t)-d_{2} \Delta p(x, t)=-a(x) p(x, t)+c \int_{\Omega} k_{2}\left(x, x^{\prime}\right) F\left(h\left(x^{\prime}, t\right)\right) p\left(x^{\prime}, t\right) d x^{\prime}+\chi_{\omega}(x) u(x, t), \quad(x, t) \in Q_{0}
$$

(with $\left.d_{2} \in(0,+\infty)\right)$ and the following boundary condition (corresponding to an isolated habitat) must be considered

$$
\partial_{\nu} p(x, t)=0, \quad(x, t) \in \Sigma_{0} .
$$

The conclusion in theorem 2.1 remains true for this new case (and follows in the same manner) if we consider $\lambda_{1}(\omega, \gamma)$ to be the solution to (1.2) corresponding to $P$, the unique nontrivial nonnegative solution to

$$
\begin{cases}-d_{2} \Delta P(x)=-a(x) P(x)+\chi_{\omega}(x) L, & x \in \Omega \\ \partial_{\nu} P(x)=0, & x \in \partial \Omega .\end{cases}
$$

Existence and uniqueness of this solution can be proved as in [2] (see also [17]).

\section{A sufficient condition for the zero-stabilization of the prey population}

Consider now the control $u \equiv L$ (corresponding to an infusion of predators at the maximum affordable level). The solution to (1.1) corresponding to this control is $\left(h^{L}, p^{L}\right)$. Since $p^{L}$ satisfies

$$
\begin{aligned}
\partial_{t} p^{L}(x, t) & =-a(x) p^{L}(x, t)+c \int_{\Omega} k_{2}\left(x, x^{\prime}\right) F\left(h^{L}\left(x^{\prime}, t\right)\right) p^{L}\left(x^{\prime}, t\right) d x^{\prime}+L \chi_{\omega}(x) \\
& \geq-a(x) p^{L}(x, t)+L \chi_{\omega}(x), \quad \text { a.e. in } Q_{0},
\end{aligned}
$$


we conclude that

$$
\liminf _{t \rightarrow+\infty} p^{L}(\cdot, t) \geq \frac{L \chi_{\omega}}{a} \quad \text { in } L^{\infty}(\Omega) .
$$

So, for any $\varepsilon>0$, there exists $T_{1}(\varepsilon)$ such that

$$
\begin{gathered}
\partial_{t} h^{L}(x, t)-d \Delta h^{L}(x, t)=r(x) h^{L}(x, t)-\frac{r(x)}{K(x)} h^{L}(x, t)^{2}+\int_{\Omega} k_{1}\left(x, x^{\prime}\right) h^{L}\left(x^{\prime}, t\right) d x^{\prime}-F\left(h^{L}(x, t)\right) p^{L}(x, t) \\
\leq r(x) h^{L}(x, t)-\frac{r(x)}{K(x)} h^{L}(x, t)^{2}+\int_{\Omega} k_{1}\left(x, x^{\prime}\right) h^{L}\left(x^{\prime}, t\right) d x^{\prime}-F\left(h^{L}(x, t)\right)\left(\frac{L}{a(x)}-\varepsilon\right) \chi_{\omega}(x)
\end{gathered}
$$

in $Q_{T_{1}(\varepsilon)}$, and using lemma A.3 in [3] we obtain that

$$
0 \leq h^{L}(x, t) \leq h_{\varepsilon}(x, t) \quad \text { a.e. in } Q_{T_{1}(\varepsilon)},
$$

where $h_{\varepsilon}$ is the solution to

$$
\begin{cases}\partial_{t} h(x, t)-d \Delta h(x, t)=r(x) h(x, t)-\frac{r(x)}{K(x)} h(x, t)^{2}+\int_{\Omega} k_{1}\left(x, x^{\prime}\right) h\left(x^{\prime}, t\right) d x^{\prime} & \\ -F(h(x, t))\left(\frac{L}{a(x)}-\varepsilon\right) \chi_{\omega}(x), & (x, t) \in Q_{T_{1}(\varepsilon)} \\ \partial_{\nu} h(x, t)=0, & (x, t) \in \Sigma_{T_{1}(\varepsilon)} \\ h\left(x, T_{1}(\varepsilon)\right)=h^{L}\left(x, T_{1}(\varepsilon)\right), & x \in \Omega .\end{cases}
$$

As in [3] it follows that

$$
\lim _{t \rightarrow+\infty} h_{\varepsilon}(\cdot, t)=H^{\varepsilon} \quad \text { in } L^{\infty}(\Omega),
$$

where $H^{\varepsilon}$ is the unique nontrivial nonnegative solution to

$$
\left\{\begin{array}{rlrl}
-d \Delta H(x) & =r(x) H(x)-\frac{r(x)}{K(x)} H(x)^{2}+\int_{\Omega} k_{1}\left(x, x^{\prime}\right) H\left(x^{\prime}\right) d x^{\prime} & & \\
-F(H(x))\left(\frac{L}{a(x)}-\varepsilon\right) \chi_{\omega}(x), & & x \in \Omega \\
\partial_{\nu} H(x)=0, & & x \in \partial \Omega .
\end{array}\right.
$$

This implies that

$$
\limsup _{t \rightarrow+\infty} h^{L}(\cdot, t) \leq M_{\varepsilon} \quad \text { in } L^{\infty}(\Omega),
$$

where $M_{\varepsilon}=E s s \sup _{\Omega} H_{\varepsilon}(x)$.

Denote $\gamma_{\varepsilon}=\min _{s \in\left(0, M_{\varepsilon}+\varepsilon\right]} \frac{F(s)}{s}$.

Theorem 3.1. If there exists $\varepsilon_{0}>0$ such that $\lambda_{1}\left(\omega, \varepsilon_{0}\right)>0$, then the prey population is zero-stabilizable. Actually, the control $u \equiv L$ stabilizes to zero the prey population.

Remark 3.2. In the case when $F(h)=\rho h$, it follows that for any $\varepsilon>0$ we have $M_{\varepsilon}=F^{\prime}(0)$. So, by theorem 3.1 we conclude that if $\lambda_{1}\left(\omega, F^{\prime}(0)\right)>0$, then the control $u \equiv L$ stabilizes to zero the prey population.

Proof. For sufficiently small $\varepsilon>0$, there exists $T_{2}(\varepsilon) \geq T_{1}(\varepsilon)$ such that $h_{\varepsilon}$ satisfies

$$
\begin{cases}\partial_{t} h(x, t)-d \Delta h(x, t) \leq r(x) h(x, t)-\frac{r(x)}{K(x)} h(x, t)^{2}+\int_{\Omega} k_{1}\left(x, x^{\prime}\right) h\left(x^{\prime}, t\right) d x^{\prime} & \\ -\gamma_{\varepsilon_{0}}\left(\frac{L}{a(x)}-\varepsilon\right) \chi_{\omega}(x) h(x, t), & (x, t) \in Q_{T_{2}(\varepsilon)} \\ \partial_{\nu} h(x, t)=0, & (x, t) \in \Sigma_{T_{2}(\varepsilon)} \\ h\left(x, T_{2}(\varepsilon)\right)=h^{L}\left(x, T_{2}(\varepsilon)\right), & x \in \Omega .\end{cases}
$$


Since $\lambda_{1}\left(\omega, \gamma_{\varepsilon_{0}}\right)>0$, it follows that $\lambda_{1}\left(\omega, \gamma_{\varepsilon_{0}}, \varepsilon\right)$, the principal eigenvalue for

$$
\begin{cases}-d \Delta \varphi(x)-r(x) \varphi(x)-\int_{\Omega} k_{1}\left(x, x^{\prime}\right) \varphi\left(x^{\prime}\right) d x^{\prime}+\gamma_{\varepsilon_{0}}\left(\frac{L}{a(x)}-\varepsilon\right) \chi_{\omega}(x) \varphi(x)=\lambda \varphi(x), & x \in \Omega \\ \partial_{\nu} \varphi(x)=0, & x \in \partial \Omega,\end{cases}
$$

is positive for sufficiently small $\varepsilon>0$ (because $\lim _{\varepsilon \rightarrow 0} \lambda_{1}\left(\omega, \gamma_{\varepsilon_{0}}, \varepsilon\right)=\lambda_{1}\left(\omega, \gamma_{\varepsilon_{0}}\right)$; the proof follows as in [4], [5]). By theorem 3.2 in [3] we conclude that $h_{\varepsilon}^{*}$, the solution to

$$
\begin{cases}\partial_{t} h(x, t)-d \Delta h(x, t)=r(x) h(x, t)-\frac{r(x)}{K(x)} h(x, t)^{2}+\int_{\Omega} k_{1}\left(x, x^{\prime}\right) h\left(x^{\prime}, t\right) d x^{\prime} & \\ \quad-\gamma_{\varepsilon_{0}}\left(\frac{L}{a(x)}-\varepsilon\right) \chi_{\omega}(x) h(x, t), & (x, t) \in Q_{T_{2}(\varepsilon)} \\ \partial_{\nu} h(x, t)=0, & (x, t) \in \Sigma_{T_{2}(\varepsilon)} \\ h\left(x, T_{2}(\varepsilon)\right)=h^{L}\left(x, T_{2}(\varepsilon)\right), & x \in \Omega,\end{cases}
$$

satisfies $\lim _{t \rightarrow+\infty} h_{\varepsilon}^{*}(\cdot, t)=0$ in $L^{\infty}(\Omega)$. Since,

$$
0 \leq h_{\varepsilon}(x, t) \leq h_{\varepsilon}^{*}(x, t) \text { a.e. in } Q_{T_{2}(\varepsilon)},
$$

we may conclude (using lemma A.3 in [3]) that $\lim _{t \rightarrow+\infty} h_{\varepsilon}(\cdot, t)=0$ in $L^{\infty}(\Omega)$ and consequently $\lim _{t \rightarrow+\infty} h^{L}(\cdot, t)=0$ in $L^{\infty}(\Omega)$.

Remark 3.3. If $F(h)=\frac{\rho h^{k}}{1+q h^{k}}$, with $k \geq 2$, then we have clariffied in the previous section that the prey population cannot be stabilized to zero.

If $k=1$, then if $\lambda_{1}\left(\omega, \gamma_{\varepsilon_{0}}\right)>0$ (for a certain $\varepsilon_{0}>0$ ) the prey population is zero-stabilizable.

Remark 3.4. If $F(h)=\frac{\rho h}{1+q h}$, then $\lim _{\varepsilon \rightarrow 0} \gamma_{\varepsilon}=F^{\prime}(0)$. By an attentive analysis of the last proof we may conclude that if $\lambda_{1}\left(\omega, F^{\prime}(0)\right)>0$, then the prey population is locally zero-stabilizable (i.e., there exists $m>0$, such that for any initial datum satisfying the hypotheses and $\left\|h_{0}\right\|_{L^{\infty}(\Omega)}<m$, there exists a control $u \in U$ such that $\lim _{t \rightarrow+\infty} h^{u}(\cdot, t)=0$ in $\left.L^{\infty}(\Omega)\right)$ and the control $u \equiv L$ stabilizes to zero the pest population (if $\left\|h_{0}\right\|_{L^{\infty}(\Omega)}$ is sufficiently small).

Remark 3.5. If the predator population diffuses in the habitat $\Omega$, then the conclusion in theorem 3.1 remains true if we consider $P$, the unique nontrivial nonnegative solution to (2.1).

Remark 3.6. If $\varphi_{1}^{*}$ is the eigenfunction corresponding to the adjoint problem for (3.2) and to $\lambda:=$ $\lambda_{1}\left(\omega, \gamma_{\varepsilon_{0}}, \varepsilon\right)$, and such that $\varphi_{1}^{*}(x)>0$ a.e. in $\Omega,\left\|\varphi_{1}^{*}\right\|_{L^{2}(\Omega)}=1$, then multiplying the first equation in (3.3) by $\varphi_{1}^{*}$ we get after an easy calculation that

$$
\frac{d}{d t}\left(\int_{\Omega} h_{\varepsilon}^{*}(x, t) \varphi_{1}^{*}(x) d x\right) \leq-\lambda_{1}\left(\omega, \gamma_{\varepsilon_{0}}, \varepsilon\right) \int_{\Omega} h_{\varepsilon}^{*}(x, t) \varphi_{1}^{*}(x) d x, \quad t>T_{2}(\varepsilon) .
$$

In conclusion

$$
\int_{\Omega} h_{\varepsilon}^{*}(x, t) \varphi_{1}^{*}(x) d x \leq \exp \left(-\lambda_{1}\left(\omega, \gamma_{\varepsilon_{0}}, \varepsilon\right) t\right) \int_{\Omega} h^{L}\left(x, T_{2}(\varepsilon)\right) \varphi_{1}^{*}(x) d x, \quad \forall t \geq T_{2}(\varepsilon) .
$$

Since there exists $M_{0} \in(0,+\infty)$ such that

$$
M_{0} \leq \varphi_{1}^{*}(x), \quad \text { a.e. } x \in \Omega
$$

(see [4]) we may infer (using (3.1) and (3.4)) that $\lim _{t \rightarrow+\infty} h^{L}(\cdot, t)=0$ in $L^{1}(\Omega)$ at the same rate as $\exp \left(-\lambda_{1}\left(\omega, \gamma_{\varepsilon_{0}}, \varepsilon\right) t\right)$. The convergence $\lim _{t \rightarrow+\infty} h^{L}(\cdot, t)=0$ in $L^{\infty}(\Omega)$ follows as in [4] (using a so called parabolic $L^{1} \rightarrow L^{\infty}$ inequality [6], [7])), at the same rate.

For sufficiently small $\varepsilon>0$ this rate is close to that of $\exp \left(-\lambda_{1}\left(\omega, \gamma_{\varepsilon_{0}}\right) t\right)$.

Remark 3.7. The results established in the last two sections also imply that the prey population is stabilizable to zero if and only if the control $u \equiv L$ stabilizes to zero the prey population. 


\section{Evaluation of the principal eigenvalue $\lambda_{1}(\omega, \gamma)$}

The deep relationship between the nonnegative zero-stabilizability of one of the components of a reactiondiffusion system and the sign of the principal eigenvalues to some related elliptic problem has been investigated in [1], [2], [4], [5] for other situations. We have seen in the previous section that the rate of stabilization to 0 of the prey population corresponding to the control $u \equiv L$ is dictated by $\lambda_{1}(\omega, \gamma)$, for a certain $\gamma\left(=\gamma_{\varepsilon_{0}}\right)$. Firstly, in order to approximate $\lambda_{1}(\omega, \gamma)$ we shall use a technique introduced by the author in [2]. For other approaches (and different situations) we refer to [11].

Consider the following particular population dynamics model with a very special logistic term

$$
\begin{cases}\partial_{t} y(x, t)-d \Delta y(x, t)=r(x) y(x, t)+\zeta y(x, t)+\int_{\Omega} k_{1}\left(x, x^{\prime}\right) y\left(x^{\prime}, t\right) d x^{\prime} & \\ -\gamma \frac{L}{a(x)} \chi_{\omega}(x) y(x, t)-y(x, t) \int_{\Omega} y(x, t) d x, & (x, t) \in Q_{0} \\ \partial_{\nu} y(x, t)=0, & (x, t) \in \Sigma_{0} \\ y(x, 0)=1, & x \in \Omega,\end{cases}
$$

where $\gamma \geq 0$ and $\zeta$ is a constant greater than $\lambda_{1}(\omega, \gamma)$.

The following result follows from theorem 4.1 in [2]:

Theorem 4.1. The solution $y^{\omega}$ to (4.1) satisfies

$$
\lim _{t \rightarrow+\infty} \int_{\Omega} y^{\omega}(x, t) d x=\zeta-\lambda_{1}(\omega, \gamma)
$$

This result provides an approximating method for $\lambda_{1}(\omega, \gamma)$ (see [2], [3]). Namely, for sufficiently large $T>0, \zeta-\int_{\Omega} y^{\omega}(x, T) d x$ approximates $\lambda_{1}(\omega, \gamma)$.

Since an important goal is to find a position for $\omega$ (in the set of all its transations) which provides a large value of $\lambda_{1}\left(\omega, \gamma_{\varepsilon_{0}}\right)$ and consequently to get a fast stabilization of the prey population via the control $u \equiv L$, it is obvious the importance of investigating the problem of finding a position for $\omega$ which gives a small value for

$$
\Phi^{\omega}=\int_{\Omega} y^{\omega}(x, T) d x
$$

Let $\omega_{0}$ be a nonempty open subset of $\Omega$, with sufficiently smooth boundary and such that $\Omega \backslash \bar{\omega}_{0}$ is a domain. Consider $\mathcal{O}$ the set of all translations $\omega$ of $\omega_{0}$, satisfying $\omega \subset \subset \Omega$. For any $\omega \in \mathcal{O}$ and $V \in \mathbf{R}^{N}$ we define the derivative

$$
d \Phi^{\omega}(V)=\lim _{\varepsilon \rightarrow 0} \frac{\Phi^{\varepsilon V+\omega}-\Phi^{\omega}}{\varepsilon} .
$$

The derivative of $\Phi^{\omega}$ with respect to translations can be evaluated as in theorem 4.2 [2]:

Theorem 4.2. For any $\omega \in \mathcal{O}$ and $V \in \mathbf{R}^{N}$ we have that

$$
d \Phi^{\omega}(V)=\gamma L \int_{0}^{T} \int_{\partial \omega} \frac{1}{a(x)} y^{\omega}(x, t) g^{\omega}(x, t) \nu(x) \cdot V d \sigma d t
$$

(here $\nu(x)$ is the normal versor at $x \in \partial \omega$, inward with respect to $\omega$ ), where $g^{\omega}$ is the solution to the adjoint problem

$$
\begin{cases}\partial_{t} g+d \Delta g+r(x) g+\int_{\Omega} k_{1}\left(x^{\prime}, x\right) g\left(x^{\prime}, t\right) d x^{\prime}-\frac{\gamma L \chi_{\omega}(x)}{a(x)} g+\zeta g & \\ \quad-\left(\int_{\Omega} y^{\omega}(x, t) d x\right) g-\int_{\Omega} y^{\omega}(x, t) g(x, t) d x=0, & (x, t) \in \Omega \times(0, T) \\ \partial_{\nu} g(x, t)=0, & (x, t) \in \partial \Omega \times(0, T) \\ g(x, T)=1, & x \in \Omega .\end{cases}
$$


This last theorem allows to derive an iterative algorithm to improve at each step the position of the domain where the control acts (by translations) in order to get a faster stabilization (via the control $u:=L)$. For details (including numerical tests) see [2].

\section{Final comments}

If we assume that the prey population is zero-stabilizable (this implies $F^{\prime}(0)>0$ ), then from Section 2 we have that $\lambda_{1}\left(\omega, F^{\prime}(0)\right) \geq 0$. On the other hand

$$
\lambda_{1}\left(\omega, F^{\prime}(0)\right) \leq \tilde{\lambda}_{1}\left(\omega, F^{\prime}(0)\right),
$$

where $\tilde{\lambda}_{1}\left(\omega, F^{\prime}(0)\right)$ is the principal eigenvalue of

$$
\begin{cases}-d \Delta \varphi(x)-r(x) \varphi(x)+\frac{L \chi_{\omega}(x)}{a(x)} F^{\prime}(0) \varphi(x)=\lambda \varphi(x), & x \in \Omega \\ \partial_{\nu} \varphi(x)=0, & x \in \partial \Omega .\end{cases}
$$

Since the elliptic operator in the left hand side is self-adjoint, we obtain via Rayleigh's principle [7] that

$$
\tilde{\lambda}_{1}\left(\omega, F^{\prime}(0)\right)=\min _{\varphi \in H^{1}(\Omega),\|\varphi\|_{L^{2}(\Omega)}>0} \frac{d \int_{\Omega}|\nabla \varphi(x)|^{2} d x-\int_{\Omega} r(x)|\varphi(x)|^{2} d x+L F^{\prime}(0) \int_{\omega} \frac{|\varphi(x)|^{2}}{a(x)} d x}{\int_{\Omega}|\varphi(x)|^{2} d x} .
$$

This implies that

$$
0 \leq \lambda_{1}\left(\omega, F^{\prime}(0)\right) \leq \frac{-\int_{\Omega} r(x) d x+L F^{\prime}(0) \int_{\omega} \frac{1}{a(x)} d x}{\int_{\Omega} d x}
$$

and consequently

$$
\int_{\omega} \frac{1}{a(x)} d x \geq \frac{1}{L F^{\prime}(0)} \int_{\Omega} r(x) d x
$$

This shows that in order to stabilize the prey population, we have to find firstly a large enough subdomain $\omega$, with a good position, in order to satisfy the last inequality.

If such a subdomain $\omega$ does not exist (this happens if for example $\left.\int_{\Omega} \frac{1}{a(x)} d x<\frac{1}{L F^{\prime}(0)} \int_{\Omega} r(x) d x\right)$, then a stabilizing control cannot exist.

Assume now that the prey population is not subject to a migration (i.e., $\left.k_{1} \equiv 0\right)$ and that $F(h)=\rho h$. Theorem 3.1 implies that if $\mu_{1}(\omega)$, the principal eigenvalue of

$$
\begin{cases}-d \Delta \varphi(x)-r(x) \varphi(x)+\frac{\rho L}{a(x)} \chi_{\omega}(x) \varphi(x)=\mu \varphi(x), & x \in \Omega \\ \partial_{\nu} \varphi(x)=0, & x \in \partial \Omega\end{cases}
$$

is positive, then the prey population is zero-stabilizable (via the control $u \equiv L$ ) and the stabilization is faster than that of $\exp \left(-\left(\mu_{1}(\omega)-\varepsilon\right) t\right)$, for any $\varepsilon>0$. On the other hand

$$
\mu_{1}(\omega) \geq \beta_{1}(\omega)
$$

where $\beta_{1}(\omega)$ is the principal eigenvalue of

$$
\begin{cases}-d \Delta \varphi(x)-r_{2} \varphi(x)+\frac{\rho L}{a_{2}} \chi_{\omega}(x) \varphi(x)=\beta \varphi(x), & x \in \Omega \\ \partial_{\nu} \varphi(x)=0, & x \in \partial \Omega .\end{cases}
$$

Here $r_{2}=\|r\|_{L^{\infty}(\Omega)}, a_{2}=\|a\|_{L^{\infty}(\Omega)}$. 
So, if $\beta_{1}(\omega)>0$, then the prey population is zero-stabilizable (via the control $u \equiv L$ ) and the stabilization is faster than that of $\exp \left(-\left(\beta_{1}(\omega)-\varepsilon\right) t\right)$, for any $\varepsilon>0$. By Rayleygh's principle we get that

$$
\beta_{1}(\omega)=-r_{2}+\min _{\varphi \in H^{1}(\Omega),\|\varphi\|_{L^{2}(\Omega)}>0} \frac{d \int_{\Omega}|\nabla \varphi(x)|^{2} d x+\frac{L \rho}{a_{2}} \int_{\omega}|\varphi(x)|^{2} d x}{\int_{\Omega}|\varphi(x)|^{2} d x} .
$$

In the particular case when $\omega$ is a ball, the maximum value of $\beta_{1}(\omega)$ in the class of all $\Omega$ of a given measure, is obtained when $\Omega$ is a ball also, and when both $\omega$ and $\Omega$ have the same center. The proof follows the lines in [1] and is based on some rearrangement techniques (see [12]):

Let $\lambda_{1}^{\Omega}(\omega)$ be the principal eigenvalue for

$$
\begin{cases}-d \Delta \varphi(x)+\frac{\rho L}{a_{2}} \chi_{\omega}(x) \varphi(x)=\lambda \varphi(x), & x \in \Omega \\ \partial_{\nu} \varphi(x)=0, & x \in \partial \Omega .\end{cases}
$$

We actually need to prove that for any domain $\tilde{\Omega} \neq \Omega$ satisfying the hypotheses of $\Omega$ and such that meas $(\tilde{\Omega})=\operatorname{meas}(\Omega)$, and for the particular case when $\omega=B\left(0 ; r_{11}\right)$ and $\Omega=B\left(0 ; r_{22}\right)$ are balls, with $0<r_{11}<r_{22}$, we get

$$
\lambda_{1}^{\Omega}(\omega)>\lambda_{1}^{\tilde{\Omega}}(\omega) .
$$

Indeed, let $\varphi^{*}$ be the eigenfunction for (5.1) corresponding to $\lambda:=\lambda_{1}^{\Omega}(\omega)$ and such that $\varphi^{*}(x)>0$ a.e. in $\Omega$ and $\left\|\varphi^{*}\right\|_{L^{2}(\Omega)}=1$.

We shall prove firstly that there exists $\tilde{M} \in(0,+\infty)$ such that $0<\varphi^{*}(x)<\tilde{M}$ a.e. in $\Omega \backslash \bar{\omega}$ and $\varphi^{*}(x)=\tilde{M}$ a.e. in $\partial \Omega$. Indeed, since the eigenspace corresponding to the eigenvalue $\lambda_{1}^{\Omega}(\omega)$ has dimension 1 we conclude that $\varphi^{*}$ has circular/spherical symmetry and so $\varphi^{*}$ satisfies (in polar coordinates)

$$
\frac{\partial^{2} \varphi^{*}}{\partial R^{2}}+\frac{1}{R} \frac{\partial \varphi^{*}}{\partial R}=-\lambda_{1}^{\Omega}(\omega) \varphi^{*}<0, \quad r_{11}<R<r_{22},
$$

where $R=\|x\|$, and consequently $\frac{\partial}{\partial R}\left(R \frac{\partial \varphi^{*}}{\partial R}\right)<0$ and so the mapping $R \mapsto R \frac{\partial \varphi^{*}}{\partial R}$ is strictly decreasing on $\left[r_{11}, r_{22}\right]$. Taking into account that for $R=r_{22}$ we get that $0=\partial_{\nu} \varphi^{*}=\frac{\partial \varphi^{*}}{\partial R}$, we may infer that $\varphi^{*}$ is strictly increasing with respect to $R$ on $\left[r_{11}, r_{22}\right]$. This yields

$$
\begin{gathered}
\lambda_{1}^{\Omega}(\omega)=\frac{d \int_{\Omega}\left|\nabla \varphi^{*}(x)\right|^{2} d x+\frac{\rho L}{a_{2}} \int_{\omega}\left|\varphi^{*}(x)\right|^{2} d x}{\int_{\Omega}\left|\varphi^{*}(x)\right|^{2} d x} \\
>\frac{d \int_{\Omega \backslash \tilde{\Omega}}\left|\nabla \varphi^{*}(x)\right|^{2} d x+\frac{\rho L}{a_{2}} \int_{\omega}\left|\varphi^{*}(x)\right|^{2} d x}{\int_{\Omega \backslash \tilde{\Omega}}\left|\varphi^{*}(x)\right|^{2} d x+\int_{\tilde{\Omega} \backslash \Omega} \tilde{M}^{2} d x}=\frac{d \int_{\tilde{\Omega}}\left|\nabla \varphi_{2}(x)\right|^{2} d x+\frac{\rho L}{a_{2}} \int_{\omega}\left|\varphi_{2}(x)\right|^{2} d x}{\int_{\tilde{\Omega}}\left|\varphi_{2}(x)\right|^{2} d x},
\end{gathered}
$$

where

$$
\varphi_{2}(x)= \begin{cases}\varphi^{*}(x), & x \in \Omega \backslash \tilde{\Omega} \\ \tilde{M}, & x \in \tilde{\Omega} \backslash \Omega\end{cases}
$$

By Rayleigh's principle we finally obtain that $\lambda_{1}^{\Omega}(\omega)>\lambda_{1}^{\tilde{\Omega}}(\omega)$ and get the conclusion.

Note that the same method may be used in order to get an upper bound for $\lambda_{1}\left(\omega, F^{\prime}(0)\right)$ and consequently and upper bound for the stabilization rate of the prey population.

Acknowledgements. This work was supported by a grant of the Ministry of National Education, CNCS-UEFISCDI, project number PN-II-ID-PCE-2012-4-0270 (68/2.09.2013): "Optimal Control and Stabilization of Nonlinear Parabolic Systems with State Constraints. Applications in Life Sciences and Economics".

The author is indebted to the referees for their valuable comments and suggestions to improve the paper. 


\section{References}

[1] B. Ainseba, S. Aniţa. Internal nonnegative stabilization for some parabolic equations. Comm. Pure Appl. Anal., 7 (3) (2008), 491-512.

[2] L.-I. Aniţa, S. Aniţa, V. Arnăutu. Internal null stabilization for some diffusive models in population dynamics. Appl. Math. Comput., 219 (20) (2013), 10231-10244.

[3] S. Aniţa. Zero-stabilization for some diffusive models with state constraints. Math. Model. Nat. Phenom., 9 (4) (2014), 6-19.

[4] S. Aniţa, V. Capasso. A stabilization strategy for a reaction-diffusion system modelling a class of spatially structured epidemic systems (think globally, act locally). Nonlin. Anal. Real World Appl. 10 (4) (2009), 2026-2035.

[5] S. Aniţa, W. Fitzgibbon, M. Langlais. Global existence and internal stabilization for a class of predator-prey systems posed on non coincident spatial domains. Discrete Cont. Dyn. Syst.- B, 11 (4) (2009), 805-822

[6] V. Barbu. Analysis and Control of Nonlinear Infinite Dimensional Systems. Academic Press, Boston, 1993.

[7] V. Barbu. Partial Differential Equations and Boundary value problems. Kluwer Academic Press, Dordrecht, 1998.

[8] V. Capasso, R.E. Wilson. Analysis of a reaction-diffusion system modelling man-environment-man epidemics. SIAM J. Appl. Math. 57 (2) (1997), 327-346.

[9] K. Deimling. Nonlinear Functional Analysis. Springer-Verlag, Berlin, 1985.

[10] S. Genieys, V. Volpert, P. Auger. Pattern and waves for a model in population dynamics with nonlocal consumption of resources. Math. Modelling Nat. Phenom. 1 (1) (2006), 65-82.

[11] A. Henrot, El Haj Laamri, D. Schmitt. On some spectral problems arising in dynamic populations. Comm. Pure Appl. Anal. 11 (6) (2012), 2429-2443.

[12] B. Kawohl. Rearrangements and Convexity of Level Sets in PDE. Springer Lecture Notes in Math. $1150,1985$.

[13] J.-L. Lions. Controlabilité Exacte, Stabilisation et Perturbation de Systemes Distribués. RMA 8, Masson, Paris, 1988.

[14] A. Okubo. Diffusion and Ecological Problems: Mathematical Models. Springer-Verlag, Berlin, 1980.

[15] J.D. Murray. Mathematical Biology. II. Spatial Models and Biomedical Applications, 3rd edition. Springer-Verlag, New York, 2003.

[16] M.H. Protter, H.F. Weinberger. Maximum Principles in Differential Equations. Springer-Verlag, New York, 1984.

[17] J. Smoller. Shock Waves and Reaction Diffusion Equations. Springer Verlag, Berlin, 1983.

[18] V. Volpert, V. Vougalter. Stability and instability of solutions of a nonlocal reaction-diffusion equation when the essential spectrum crosses the imaginary axis. Preprint (2014), https : //www.ma.utexas.edu/mparc/index-14.html. 